\title{
Preliminary Analysis of Physiological Changes of Nursing Students during Training
}

\author{
Mladen Milosevic, Member, IEEE, Emil Jovanov, Senior Member, IEEE, \\ Karen H. Frith, Julie Vincent, Eric Zaluzec \\ University of Alabama in Huntsville, Huntsville, Alabama, U.S.A
}

\begin{abstract}
Long-term exposure to stress has been associated with chronic diseases, depression, and immune disorders. The precise detection and assessment of stress depends on personalized physiological monitoring and assessment of influence of personal and workplace factors We monitored nursing students during training on a high fidelity simulator in the Real-time Physiological Monitoring Lab at the University of Alabama in Huntsville. In this paper we present the preliminary results of this pilot study. A total of 14 participants were recorded: 12 female and 2 male subjects, 23-46 years old with an average age of 32.8 years. We analyzed heart rate, Heart Rate Variability (HRV), respiration, and physical activity. The results indicate significant strain on subjects during simulation: heart rate increased $16.7 \%$, from 82.8 to $96.6 \mathrm{bpm}(\mathrm{p}<0.001)$, falling to a slightly increased level after the training session (84.9 bpm); Root Mean Square of Successive RR Differences (RMSSD) decreased from $38.9 \mathrm{~ms}$ to $37.7 \mathrm{~ms}$; the breathing rate increased during the simulation from 16.9 to 17.7 breaths/min. Distractions also significantly influenced physiological parameters: the first telephone call increased heart rate on average $9 \mathrm{bpm}(\mathrm{p}<0.001)$, while the second call increased heart rate $8.6 \mathrm{bpm}(\mathrm{p}<0.001)$. The simulated patient-related events created even more prominent response; the average heart rate increased $17.4 \mathrm{bpm}(\mathbf{p}<\mathbf{0 . 0 0 1})$ at the onset of "patient in crisis" event. Real-time wearable monitoring may provide assessment of occupational stress.
\end{abstract}

\section{INTRODUCTION}

The work of nurses is inherently stressful. Nurses care for patients at the most vulnerable points in their liveswaking after surgery with intense pain, losing the battle with cancer, being readmitted for heart failure, and many other difficult situations. Not only do patient situations demand the cognitive attention of nurses, but they also require the completion of numerous tasks. Lindberg et al. [1] found that registered nurses on average complete over 160 tasks during 8 hours (2.5 minutes per task). Nurses are constantly interrupted by telephone calls, beeps, and alarms; Hall et al. [2] observed over 13,000 disruptions in the work of 360

This work was supported in part by the University of Alabama in Huntsville under Grant UAH234343.

E. Jovanov is with the Electrical and Engineering Department, University of Alabama in Huntsville, Huntsville, AL 35899 (Phone: 256824-5094; fax: 256-824-6803; e-mail: emil.jovanov@uah.edu).

K. Frith and Julie Vincent are with the College of Nursing, University of Alabama in Huntsville, Huntsville, AL 35899, USA (e-mail: karen.frith@uah.edu and jav0004@uah.edu).

M. Milosevic and Eric Zaluzec are with the Electrical and Computer Engineering Department, University of Alabama in Huntsville, Huntsville, AL 35899 (e-mail: mladen.milosevic@uah.edu and ejz0060@uah.edu ). nurses in a 2 -week period. Solomon et al. [3] found that interruptions comprised over $60 \%$ of the factors contributing to workplace stress in hospitals. This un-relenting demand on nurses causes stress and strain. Salmond and Ropis [4] found that stress caused by frequent interruptions impacts the general health and well-being of nurses.

Although stress can increase the mental acuity of nurses and readiness to intervene when patient conditions deteriorate, long-term stress is harmful to the physical and mental health of nurses. Such consequences include heart disease, high blood pressure, depression, difficulty sleeping, and anxiety [5]. McEwen [6] describes a phenomenon called "allostasic overload," which explains some of the physiological results of long-term stress. McEwen explains that under situations that induce short-term stress in individuals, the physiological parameters that indicate stress, heart rate variability and blood pressure, return to their baseline. In contrast, under multiple short-term stressful events or long-term stress, the physiological parameters do not return to baseline, but rather establish a new baseline that can result in chronic health problems. Explained at a more cellular level, the hypothalamic-pituitary-adrenal and sympathetic-adrenalmedullary systems respond to stress, keeping the body ready to respond quickly to changing situation [7][8]. Over long periods of time these physiological process are responsible for chronic physical illnesses [9].

Appelhans and Luecken describe two theories (polyvagal theory and neurovisceral integration) that connect emotional responses to the activation of the autonomic nervous system and cardiac activity, specifically heart rate variability (HRV) [10]. In general, in healthy emotional responses to social situations, HRV is greater; however, reduced HRV is seen when coping is insufficient to the demands of the situation. This insufficient coping results in anxiety or depression. Studies of occupational stress have shown that HRV is a reliable measure indicative of strain from insufficient coping to stress [11][12][13]. Early detection of "allostasic load" and interventions to reduce the strain responses could interrupt the progression to chronic illness.

From an organizational point of view, occupational stress of nurses is an important factor because of its relationship to employee productivity and performance. Occupational stress can lead to absenteeism, burnout, and turnover [14]. These consequences of chronic stress are not only harmful to healthcare organizations where nurses work, but they affect 
patients. High stress in the hospital work environment has a strong correlation to increased numbers of patient care errors according to Dugan et al. [15]. When a sufficient number of stressed nurses work together, the satisfaction of patients on that hospital unit suffers [16].

The precise assessment of stress depends on personalized physiological monitoring and assessment of influence of personal and workplace factors. Promising tools in stress research are wearable sensors that can record continuous heart rate, blood pressure, and heart rate variability. When recordings of physiological parameters are paired with subjective measurements of stress, new information about intermediate indicators of strain can be developed.

In order to detect and assess stress, many researchers approached inducing stress in the participants of the study. For this purpose they used many different methods, from mental arithmetic [17][18], memory tests [19][20], and puzzles [21], audio and movie stimulants [22] to electric shocks [23]. However, we chose to simulate a hospital work environment as the means of inducing stress in participants.

The purpose of the current study was to examine the physiological responses of registered nurses during nursing care on a high-fidelity patient simulator, and to identify the best physiological parameters suitable for detecting occupational stress during simulated nursing activities. Many physiological parameters were recorded and calculated during stress related studies including, such as ECG, heart rate, blood pressure, respiration, skin conductance, skin temperature, and voice. In this paper we present the results of monitoring of changes in heart rate, HRV, respiration, and physical activity.

\section{METHODS}

In this study we conducted monitoring of nursing students during training on a high-fidelity patient simulator. All experiments were conducted in the Real-time Physiological Monitoring Lab [24], which is collaboratively run by the College of Engineering and College of Nursing at the University of Alabama in Huntsville.

\section{Experimental setup}

We used a control room with a one-way mirror and a private patient room equipped with high fidelity simulator, functional headwall, state of the art physiological monitors, and audio and video monitoring systems. From the control room, we ran the simulated nursing activity, and using our custom software synchronously recorded the activity of the participant from a video camera and their physiological signals from a Zephyr BioHarness 3, placed as chest belt [25]. All records of the physiological signals are automatically uploaded to the research server. The records contain interbeat intervals, heart rate, breathing rate, and acceleration on chest belt. BioHarness samples ECG at $250 \mathrm{~Hz}$ and on-belt accelerometer at $100 \mathrm{~Hz}$. A nursing student during a training session on the high fidelity patient simulator in the Real-time Physiological Monitoring Lab is shown on the Figure 1.

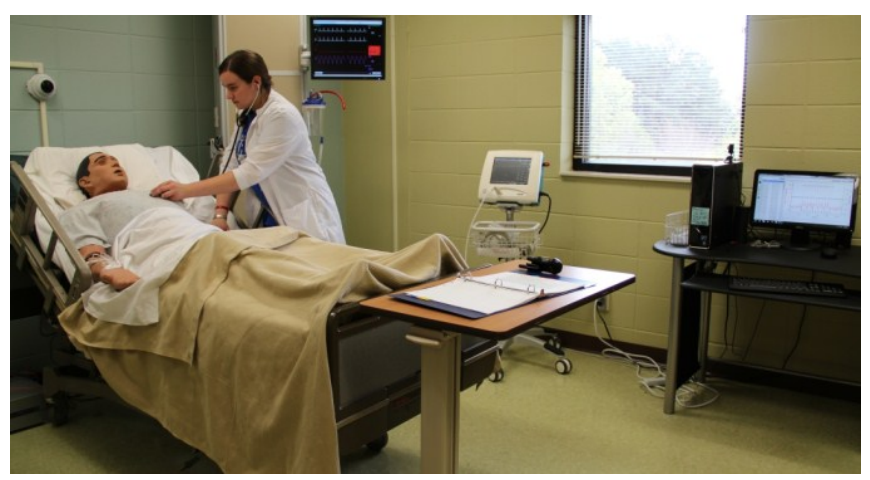

Figure 1. Training session in the Real-time Physiological Monitoring Lab

\section{Participants}

We used a non-probability sampling method to obtain research participants from students enrolled in either the Bachelor of Science in Nursing program or the Master of Science in Nursing program. Only registered nurses could participant in the study due to the type of simulation that was used.

We excluded participants on the basis of three criteria: previous diagnosis of high blood pressure or irregular heart rhythm, latex allergy, or age less than 19 years. During online registration, we collected demographic data from the volunteers.

A total of 14 participants were recorded: 12 female and 2 male subjects. The mean age of participants was 32.8 years old, with the youngest participant being 23 and the oldest participant being 46 years old.

\section{Signal Processing}

Typical parameters of Heart Rate Variability (HRV) are calculated from RR intervals on 60 second window with 30 second overlap using BioSig for Matlab [26]. A small window size is selected to support future real-time monitoring and warning system implementation. RR intervals are filtered using Kubios HRV analysis software [27]. Analyzed parameters include Root Mean Square of Successive RR Differences (RMSSD), spectral power in Low Frequency (LF) and High Frequency (HF) bands using autoregressive (AR) and Fast Fourier Transform (FFT) methods [28]. Statistical significance was estimated using t-test with $\mathrm{p}<0.05$.

Average acceleration magnitude is calculated on raw (unfiltered) data to assess physical activity of participants.

\section{Simulation protocol}

We developed a simulation that required participants to care for a patient with a tracheostomy used for breathing. We used a computer program to run the simulation in exactly the same order for each participant. The simulation protocol ran for 30 minutes for every participant encounter and included the following elements:

- Measurement of baseline physiological participant data for 5 minutes 
- Initiation of simulation with report from off-going nurse about patient's condition from the night shift

- Assessment of patient by the participant

- Interruption of respiratory assessment by telephone call to participant (Call \#1)

- Documentation of assessment findings by participant

- Interruption of documentation by telephone call to participant (Call \#2)

- "Patient in crisis" - sudden respiratory distress of the simulated patient (coughing, difficulty breathing, low oxygen saturation, elevated heart rate and blood pressure that required urgent intervention by the participant)

- Concern from family member at bedside (questioning nurse and verbalizing fear)

- End of simulation when participant successfully treats the mucous plug by pre-oxygenation and suctioning

- Measurement of participants' physiological data for 5 minutes after the simulation.

The experimental protocol was approved by the UAHuntsville IRB committee (FWA00016967).

\section{RESULTS}

A summary of the selected physiological parameters for all subjects is presented in Table 1. We used repeated measures analysis of variance to evaluate changes in physiological data during before, during, and after events in the simulation; the results are described in the paragraphs below.

The training session creates significant strain on subjects. On average, heart rate increased $16.7 \%$, from 82.8 to 96.6 bpm $(\mathrm{p}<0.001)$, falling to a slightly increased level after the training session (84.9 bpm). All subjects had increased heart rate even before the training session that can be attributed to anxiety in anticipation of the session. For example, subject \#5 had average heart rate before the session $108.9 \mathrm{bpm}$, $125.8 \mathrm{bpm}$ during session, and $108 \mathrm{bpm}$ after the session. Heart rate changes during training session are shown on Figure 2, and presented values are minimum, average, and maximum for all subjects. The prolonged effects of the stress are visible in higher heart rate and lower heart rate variability (e.g. RMSSD decreased from $38.9 \mathrm{~ms}$ to $37.7 \mathrm{~ms}$ ). The breathing rate increased also during the simulation from 16.9 to 17.7 breaths $/ \mathrm{min}$.

Physical activity did not significantly influence heart rate during the session, as observed from bursts of dynamic magnitude of 3D acceleration. Average 3D acceleration during simulation is only $0.1 \mathrm{~g}$ while before and after simulation is $0.04 \mathrm{~g}$ (Table 1 ).

The ratio of the low-frequency and high-frequency components of the RR spectrum (LF/HF) significantly changes during the simulation from 2.16 to $4.68(\mathrm{p}<0.001)$ and follows the same pattern as heart rate - increases during the simulation and slowly returns to normal after the session $2.4(\mathrm{p}<0.001)$.

The simulated patient-related events (e.g. "patient in crisis") create significant physiological responses. The average heart rate increases $17.4 \mathrm{bpm}(\mathrm{p}<0.001)$ at the onset of the event. It is interesting to note that subjects express emotional response even in the case of the "automated dummy" (high fidelity simulator). It should be noted that during "patient in crisis" event average 3D acceleration magnitude is only $0.03 \mathrm{~g}$, which is lower than quiet state before the simulation. Therefore, increase in HR is mostly stress related, rather than induced by physical activity.

TABLE I. SUMMARY OF THE SELECTED PHYSIOLOGICAL PARAMETERS FOR ALL SUBJECTS; MEAN \pm STANDARD DEVIATION

\begin{tabular}{|l|c|c|c|c|c|c|}
\hline \multicolumn{1}{|c|}{ Parameter } & $\begin{array}{c}\text { Before } \\
\text { Simulation }\end{array}$ & $\begin{array}{c}\text { During } \\
\text { Simulation }\end{array}$ & $\begin{array}{c}\text { After } \\
\text { Simulation }\end{array}$ & $\begin{array}{c}\Delta \text { for Call } \\
\# \mathbf{1}\end{array}$ & $\begin{array}{c}\boldsymbol{\Delta} \text { for Call } \\
\# \mathbf{2}\end{array}$ & $\begin{array}{c}\Delta \text { for Patient in } \\
\text { Crisis }\end{array}$ \\
\hline HR[bpm] & $82.76 \pm 12.29$ & $96.61 \pm 14.01$ & $84.85 \pm 14.86$ & $9 \pm 7.99$ & $8.63 \pm 6$ & $17.38 \pm 10.39$ \\
\hline RMSSD [ms] & $38.86 \pm 21.2$ & $29.81 \pm 13.6$ & $37.65 \pm 18.65$ & $-2.84 \pm 9.76$ & $3.64 \pm 12.18$ & $-7.06 \pm 20.98$ \\
\hline AR LF/HF & $2.16 \pm 1.77$ & $4.68 \pm 2.69$ & $2.4 \pm 1.48$ & $-0.97 \pm 3.47$ & $0.14 \pm 4.74$ & $1.66 \pm 6.02$ \\
\hline FFT LF/HF & $2.36 \pm 2.62$ & $5.18 \pm 3.04$ & $2.42 \pm 1.41$ & $-0.64 \pm 5.17$ & $-2.15 \pm 9.9$ & $1.34 \pm 7.86$ \\
\hline Breathing rate [bpm] & $16.88 \pm 2.24$ & $17.73 \pm 3.72$ & $16.79 \pm 4.34$ & $0.9 \pm 3.05$ & $-0.75 \pm 5.11$ & $0.69 \pm 3.53$ \\
\hline Acceleration Magnitude[g] & $0.04 \pm 0.02$ & $0.1 \pm 0.01$ & $0.04 \pm 0.02$ & $0.02 \pm 0.05$ & $0.01 \pm 0.04$ & $0.03 \pm 0.06$ \\
\hline
\end{tabular}

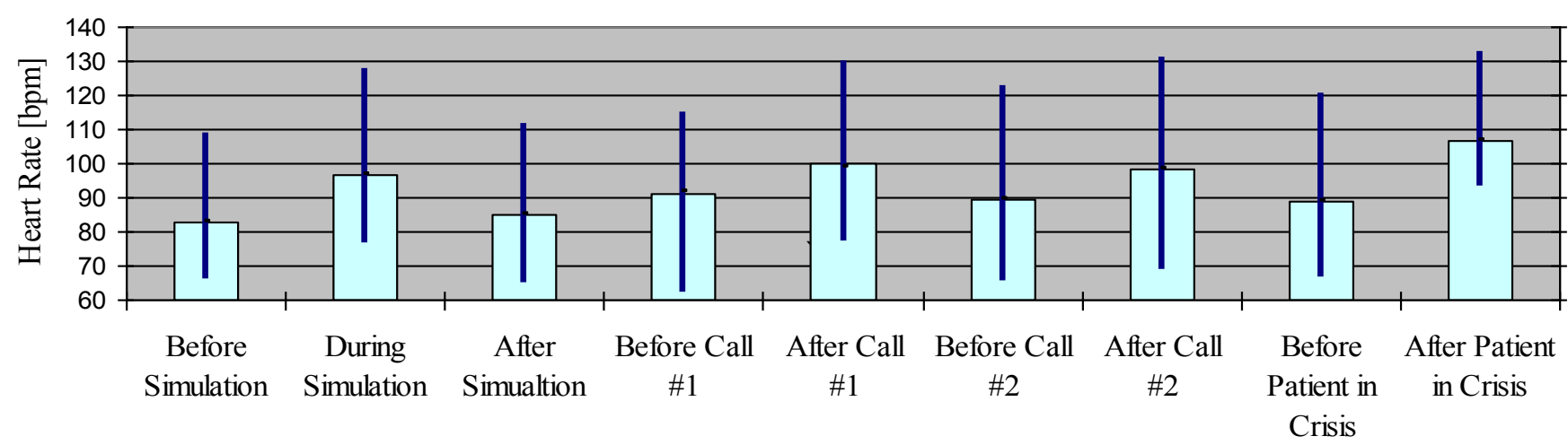

Figure 2. Heart rate changes during training session; Minimum/Average/Maximum for all subjects 
Other disturbances also significantly influence physiological parameters by increasing demands in already stressful situation. We used telephone calls to distract the participants during the training session. The first telephone call increased heart rate on average $9 \mathrm{bpm}(\mathrm{p}<0.001)$, while the second call increased heart rate $8.6 \mathrm{bpm}(\mathrm{p}<0.001)$.

Transient nature of event-related changes probably leads to inconclusive changes of RMSSD and spectral parameters as presented in Table 1 .

\section{Discussion AND CONCLUSION}

Real-time wearable monitoring of occupational stress of nurses or nursing students may facilitate objective assessment of physiological changes and facilitate collection of subjective responses about the source of stress in the workplace.

In this paper we presented the preliminary results of a pilot study conducted during training of nursing students in the Real-time Physiological Monitoring Lab. Although physiological parameters differed from participant to participant they all exhibited similar patterns in response to the events. It was surprising how much work place disturbances, such as telephone calls, caused changes in the physiological parameters.

Results of this study will be used to define signal processing methods for the optimal assessment of strain during training.

\section{ACKNOWLEDGMENT}

We are grateful to Ms. Greer Eleazer, Director of the Learning Resource Center in the College of Nursing, University of Alabama in Huntsville for help with organization of experiments and participant recruitment.

\section{REFERENCES}

[1] C. Lindberg, S. Nash, and C. Lindberg, Nurses Take Note: A Primer on Complexity Science. On the Edge: Nursing in the Age of Complexity. Bordentown, NJ: Plexus Press, 2008.

[2] L. M. Hall, M. Ferguson-pare, E. Peter, D. White, J. Besner, A. Chisholm, E. Ferris, M. Fryers, M. Macleod, B. Mildon, C. Pedersen, and A. Hemingway, "Going blank: factors contributing to interruptions to nurses' work and related outcomes," Journal of Nursing Management, vol. 18, no. 8, pp. 1040-1047, Nov. 2010.

[3] D. Solomon, N. Albert, Z. Sun, A. M. Bowers, and M. Molnar, "Complexity of Care Is Associated With Distressing Environmental Factors," Clinical Nurse Specialist, vol. 25, no. 4, pp. 186-192 10.1097/NUR.0b013e318221f2d3, 2011.

[4] S. Salmond and P. Ropis, "Research for practice. Job stress and general well-being: a comparative study of medical-surgical and home care nurses.," MEDSURG NURS, vol. 14, no. 5, pp. 301-309, Oct. 2005.

[5] A. McVicar, "Workplace stress in nursing: a literature review," Journal of Advanced Nursing, vol. 44, no. 6, pp. 633-642, 2003.

[6] B. S. McEwen, "Stressed or stressed out: what is the difference?," $J$ Psychiatry Neurosci, vol. 30, no. 5, pp. 315-318, Sep. 2005.

[7] E. McNeely, "The consequences of job stress for nurses' health: Time for a check-up," Nursing Outlook, vol. 53, no. 6, pp. 291-299, Nov. 2005.

[8] J. Sun, S. Wang, J.-Q. Zhang, and W. Li, "Assessing the cumulative effects of stress: The association between job stress and allostatic load in a large sample of Chinese employees," TWST, vol. 21, no. 4, pp. 333-347, Oct. 2007.

[9] D. C. Ganster, "Measurement challenges for studying work-related stressors and strains," Human Resource Management Review, vol. 18, no. 4, pp. 259-270, Dec. 2008.

[10] B. M. Appelhans and L. J. Luecken, "Heart rate variability as an index of regulated emotional responding.," Review of General Psychology, vol. 10, no. 3, pp. 229-240, 2006.

[11] S. M. Collins, "Emerging methods for the physiological assessment of occupational stress," Work, vol. 17, no. 3, pp. 209-219, 2001.

[12] L. C. Gallo, L. M. Bogart, A.-M. Vranceanu, and L. C. Walt, "Job characteristics, occupational status, and ambulatory cardiovascular activity in women," Ann Behav Med, vol. 28, no. 1, pp. 62-73, Aug. 2004.

[13] Rajiv Kumar and Naveen K. Goel, "Current Status Of Cardiovascular Risk Due To Stress," The Internet Journal of Health, vol. 7, no. 1, 2008.

[14] M. M. Davey, G. Cummings, C. V. Newburn-Cook, and E. A. Lo, "Predictors of nurse absenteeism in hospitals: a systematic review," $J$ Nurs Manag, vol. 17, no. 3, pp. 312-330, Apr. 2009.

[15] J. Dugan, E. Lauer, Z. Bouquot, B. K. Dutro, M. Smith, and G. Widmeyer, "Stressful Nurses: The Effect on Patient Outcomes," Journal of Nursing Care Quality, vol. 10, no. 3, 1996.

[16] A. N. Garman, P. W. Corrigan, and S. Morris, "Staff burnout and patient satisfaction: evidence of relationships at the care unit level," $J$ Occup Health Psychol, vol. 7, no. 3, pp. 235-241, Jul. 2002.

[17] G. Krantz, M. Forsman, and U. Lundberg, "Consistency in physiological stress responses and electromyographic activity during induced stress exposure in women and men," Integrative Physiological and Behavioral Science, vol. 39, no. 2, pp. 105-118, Apr. 2004.

[18] X. Yu, J. Zhang, D. Xie, J. Wang, and C. Zhang, "Relationship between scalp potential and autonomic nervous activity during a mental arithmetic task," Auton Neurosci, vol. 146, no. 1-2, pp. 8186, Mar. 2009.

[19] N. Hjortskov, D. Rissén, A. K. Blangsted, N. Fallentin, U. Lundberg, and K. Søgaard, "The effect of mental stress on heart rate variability and blood pressure during computer work," Eur. J. Appl. Physiol., vol. 92 , no. 1-2, pp. 84-89, Jun. 2004.

[20] A. K. Blangsted, K. Søgaard, H. Christensen, and G. Sjøgaard, "The effect of physical and psychosocial loads on the trapezius muscle activity during computer keying tasks and rest periods," Eur. J. Appl. Physiol., vol. 91, no. 2-3, pp. 253-258, Mar. 2004.

[21] F. W. Kok, H. G. Westenberg, J. H. Thijssen, and J. M. van Ree, "Endocrine and cardiovascular responses to a series of graded physical and psychological stress stimuli in healthy volunteers," Eur Neuropsychopharmacol, vol. 5, no. 4, pp. 515-522, Dec. 1995.

[22] B. Grundlehner, L. Brown, J. Penders, and B. Gyselinckx, "The Design and Analysis of a Real-Time, Continuous Arousal Monitor," 2009, pp. 156-161.

[23] J. T. Noteboom, K. R. Barnholt, and R. M. Enoka, "Activation of the Arousal Response and Impairment of Performance Increase with Anxiety and Stressor Intensity," J Appl Physiol, vol. 91, no. 5, pp. 2093-2101, 2001.

[24] "Real-time Physiological Monitoring Laboratory." [Online]. Available: http://www.ece.uah.edu/ jovanov/labs/RTPLab/. [Accessed: 24-Mar-2012].

[25] "Zephyr BioHarness BT." [Online]. Available: http://www.zephyrtechnology.com/bioharness-bt. [Accessed: 28-Mar-2012].

[26] "BioSig: A biosignal processing toolbox for Octave and Matlab." [Online]. Available: http://biosig.sourceforge.net/projects.html. [Accessed: 07-Jun-2012].

[27] M. P. Tarvainen, J.-P. Niskanen, J. A. Lipponen, P. O. Ranta-aho, and P. A. Karjalainen, "Kubios HRV - A Software for Advanced Heart Rate Variability Analysis," in 4th European Conference of the International Federation for Medical and Biological Engineering, vol. 22, J. Sloten, P. Verdonck, M. Nyssen, J. Haueisen, and R. Magjarevic, Eds. Springer Berlin Heidelberg, 2009, pp. 1022-1025.

[28] M. Malik, "Heart Rate Variability, Standards of Measurement, Physiological Interpretation, and Clinical Use," European Heart Journal, vol. 17, pp. 354-381, 1996. 